\title{
Kirche und Medien: die Welt als Dorf
}

\author{
von Frans Oudejans
}

Ein Dorf ohne Eltern, so hat der belgische Priesterdichter Anton van Wilderode einen Ort genannt, an den er nach langer Zeit zurückkehrte. Das Vertraute schien verschwunden zu sein: das Dorf war ganz und gar in Fertigware verwandelt: mit Reihenhäusern, Einheitsläden, neuen Straßenzügen und Ampeln. Was ihm einst seine Atmosphäre gegeben hatte, war nicht mehr.

Solch ein modernes Dorf wäre die Welt, wenn die Gelehrten Recht behalten hätten, die vornehmlich in den idealistischen sechziger Jahren ein Weltdorf („global village") voraussagten, eine Welt, die nur noch ein einziges großes Dorf wäre. Die Technik würde es möglich machen, daß jeder mit jedem mühelos in Kontakt treten könnte, die Sorge für einander würde auf der ganzen Erde zu Entwicklung und Wohlstand führen, und jeder würde sogar das Wort vom Mund des anderen ablesen. Ein Dorf also, in dem all unsere Lebensäußerungen miteinander verflochten wären, und das planetenweit.

Von dieser Vorhersage ist inzwischen eine Menge eingetroffen, mehr als wir auf den ersten Blick begreifen. Das gilt jedenfalls für die technische Machbarkeit, die Möglichkeit der Kommunikation. In der Tat gehören Safarireisen nach Kenia und Trips nach Bangkok oder Mexiko heute zu der Art und Weise, wie man seine Ferien verbringt. Und innerhalb kürzester Zeit wissen wir, wie die Wahlen in Israel oder die Gipfelbesprechungen auf den Bermudas verlaufen sind. Ein Aufstand in El Salvador oder ein Mordanschlag auf einen Popsänger werden uns auf der Stelle bekannt. Nicht umsonst laufen die Nachrichten über den Rundfunk jetzt rund um die Uhr bei Tag und bei Nacht. Wer bereits über Teletext verfügt, bekommt die Nachrichten sogar noch schneller auf seinen Bildschirm, auf Abruf.

Wir bekommen Bilder in einem nicht zu überbietenden Tempo: den neuen Präsidenten, die Rebellion, den niedergeschossenen Sänger, das Allerneueste dank Farbfernsehen, sogar das Blut, alles.

Eine heute noch nicht einmal so alte Altersgruppe mußte eine Woche oder noch länger auf die Wochenschau warten, wir aber erleben die Zeit gerafft. Wo einst Wochen, manchmal Monate nötig waren, um einem anderen Weltteil einen Bericht zu übermitteln, geschieht das jetzt in Sekunden. Wir setzen Satelliten dafür ein. Daß Entwicklung und Wohlstand nicht auf Weltebene eingetreten sind, hat die Spannungen in der Welt jedoch erhöht. Iran ist für das ferne Amerika von Bedeutung, und die Sowjetunion beobachtet, wie Peru sich entwickelt.

Die Welt, die zum Dorf wird, hat keine Eltern. Sie hat höchstens Vormünder. Die Verfremdung, die sie dank der verbesserten Kommunikationstechniken gerade überwinden sollte, erscheint größer denn je. Sogar in dem begrenzten Gebiet der Niederlande mit seinen 33000 Quadratkilometern nimmt die Kluft zwischen „echten ${ }^{\text {“ Nie- }}$ derländern und Gruppen zu, die aufgrund der geschrumpften Entfernungen in der

Frans Oudejans ist Pressereferent des Bistums Breda, Niederlande, und befaßt sich seit langen Jahren mit der Praxis und Theorie der kirchlichen publizistischen Árbeit. 
Welt hereinkamen, gebeten oder ungebeten. Wie können wir uns auch noch sorgen um Kambodschaner, Argentinier und Bantus andernorts, wenn eine vielrassige Gesellschaft bereits so mühsam $z u$ sein scheint.

Wir leben, der eine mehr als der andere, in einer Ich-Periode, in der Rufe erklingen wie "Vom neuen Verwöhnen", "Gönn dir selbst was" und "Wenn ich nur glücklich bin". Das ist die Unlogik einer Entwidklung, in der Kommunikation in das Gegenteil umzuschlagen scheint.

Lange dachte man, daß die Anhäufung von Elend, über das Fernsehen sichtbar gemacht, den Menschen ein Gefühl von Solidarität oder Schuld beigebracht hätte: „Da müssen wir doch etwas tun." Später kam eine Form von Ohnmacht hinzu unter dem Motto: „Es ist ja wirklich schlimm, aber ich kann doch nicht alles." Es entstand die große Chance für Wohltätigkeitsaktionen über Fernsehen zum Nutzen von Behinderten, Leprakranken, Hungernden in Indien.

„Wenn man genügend Elend zeigt, öffnen sich die Geldbörsen, denn die Menschen sind so schlecht noch nicht!" hieß es damals. Auch das scheint vorbei zu sein: Jetzt sind Glückspiele nötig, die den guten Gebern selbst ein Auto oder einen Geldbetrag bescheren, wenn man der dritten oder einer anderen Welt damit nützen will. Eigeninteresse unter der Maske der Mildtätigkeit. Noch vor Ablauf dieses Jahrhunderts braucht man nicht einmal mehr vor die Tür, um ein Formular für eine Wohltätigkeitsaktion zu holen. Das besorgt man dann elektronisch von daheim, also mit noch weniger sozialem Kontakt. Zu der Zeit erfolgt dann hoffentlich wieder ein Umschlag, denn so schlecht ist die Menschheit nun auch wieder nicht ...

Tatsächlich: noch immer stehen Menschen auf, die gegen den Strich und den Zeitgeist anzugehen wagen. Menschen, die sich einsetzen für eine bessere, oder zumindest eine teilweise bessere Welt. Wir begegnen ihnen überall, in manchen politischen Parteien, in allen möglichen Aktionen wie bei denen, die gegen Kernwaffen oder für die Befreiung in Südafrika sind. Menschen, die hinweisen auf Unrecht, Auseinandersetzung und Armut und etwas dagegen unternehmen wollen, Rufende in der Wüste oft, brotlose Propheten.

Diese Menschen finden wir überall, sowohl unter Älteren als unter Jüngeren. Man findet sie glücklicherweise in nicht geringem $\mathrm{Maß}$ auch im Christentum, das noch immer viele inspiriert, gegen die Not anzugehen und sich mit Herz und Seele für die Leidenden einzusetzen. Jeder kann froh sein, wenn er sich gerade in dieser Zeit als Christ gerufen weiß, die Möglichkeiten dieser Welt zu nutzen. Auch wenn er für andere „ein Argernis und eine Torheit ${ }^{*}$ ist $^{1}$.

\section{II}

Noch nie ist das Leben so nervenaufreibend gewesen. Gerade die Tatsache, daß wir alles übereinander wissen können, bietet ungeahnte Möglichkeiten.

Innerhalb der Kirchen wird die Bedeutung der (neuen) Medien oft besser begriffen als in die Praxis umgesetzt. Dabei spielt sowohl das Gefühl der Ohnmacht ebenso eine Rolle, wie auch der echte Wille, ihnen den Vorrang einzuräumen.

Der schönen Worte gibt es genug: vom Zweiten Vatikanischen Konzil (1962-1965) vom Weltrat der Kirchen (Uppsala 1968), von nationalen und lokalen Synoden und Konferenzen. Die sechziger Jahre waren großzügiger damit als die Zeit danach. Viel wurde in der Tat damals bereits gesagt. Es war nicht nur die Zeit des Glaubens an eine neue, bessere Welt, sondern auch die Zeit der Entdeckung neuer Mittel. Oft gingen Bischöfe und andere Kirchenführer von der wenig konstruktiven Devise aus „Wir 
müssen lernen damit zu leben", aber sie taten zumindest etwas dafür. Der Schwung nutzte sich $a b$ und wirkte nicht weiter: der rückläufige Bestand an Geistlichen, der Raubbau, der mit den Verbleibenden getrieben wurde (und wird), und interne Streitereien waren nicht geeignet, um Theorie in die Praxis umzusetzen. Vor langer Zeit waren die Kirchen in Europa einmal die tatsächlichen Alleinherrscher der gesellschaftlichen Kommunikation. Das ist für immer vorbei: „Die Kirchen sind eine jener Gruppen geworden, die sich einen Platz in den Medien zu erobern trachten. Sie werden mehr und mehr lernen müssen, in einer offenen Situation zu leben, in der ihre Botschaft eher Gewicht bekommt durch eigene Ursprünglichkeit und den Wahrheitsgehalt dessen, was sie sagen und tun, als durch diese oder jene Autorität ... Vielleicht hat die Tatsache, daß die Kirchen den Medien als Überbringer fremder Werte mißtrauten, es ihnen leichter gemacht, sich der Verantwortung der Sorge für eine gerechte Entwicklung zu entziehen .... Zu oft gebrauchen die Kirchen diese Auslaßmöglichkeit als erweiterte Form ihrer Kanzeln und Pfarrblätter, und was sie produzieren, entspricht oft ganz und gar nicht den Maßstäben der Integrität und der Fachkenntnis. Wo Kirchen zu tun haben mit anderer als religiöser Kommunikation, liefern sie oft eine schlechte Imitation dessen, was andere besser tun ${ }^{\alpha 3}$.

Es ist dringend eine Theologie der Massenmedien nötig, sagte mir einmal jemand, denn es hat noch niemand wirklich klar erkannt, was ihr damit tun könnt. Theologische Betrachtungen über Christus als Kommunikator par excellence sind zwar gut, reichen aber nicht viel weiter als jene Theologen, die ihre eigenen Wege gehen, und erreichen selten oder nie jene Menschen in den Medien, auf die es zuallererst ankommt, sofern sie iberhaupt etwas davon begreifen ${ }^{4}$. Solange die Kirche besteht, betreibt sie Kommunikation. Kein geringerer als der erste Papst schrieb bereits Briefe an seine Gemeinde. Sein Bischofskollege Paulus strebte eine faßbare Sprache an wenn Petrus das auch bisweilen noch schwierig fand - , und er schrieb die Stïcke, die er für besonders wichtig hielt, in größeren Buchstaben (heute heißt das in der Fach-

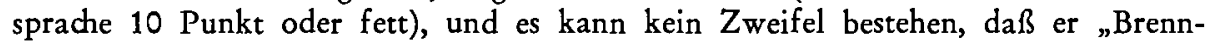
punkte $^{\star}$ oder eine andere Nachrichtenrubrik nicht ausgeschlagen hätte, wenn ihre Vertreter ihn während einer seiner Reisen aufgesucht hätten ${ }^{5}$. Und in der Tat, wie könnte ein Papst urbi et orbi, zur Stadt und der Welt, ohne Medien sprechen? „Ihr seid unsere Stimme, nicht nur die Träger unserer Stimme, sondern unsere Stimme selbst; denn wie wenige würden unsere Stimme vernehmen, wäre es nicht durch euch" .

Eine alte Neuigkeit ist das also. Das Christentum fuhr und fährt so schlecht mit der Kommunikation nicht, und es ist oft recht hübsch, dazu zu gehören und dabei mittun zu können. Aber es ist leichter gesagt als getan, eine Ergänzung, ja einen Ersatz für ehemalige Ausdrucksmittel unserer Religion wie Kult, Predigt und Glockengeläut in der Form von Zeitung, Rundfunk und Fernsehen zu finden. Die ersten drei hat man selbst in der Hand, sogar ohne Einspruch. Bei der zweiten Gruppe ist man nicht nur abhängig von anderen, sondern auch von völlig eigenen Erfordernissen. Das sind die Fertigkeiten, die auch noch je nach Medium unterschieden sind: manche TV-Persönlichkeit erweist sich als ein armseliger Zeitungsschreiber, und eine ganze Reihe Rundfunkmenschen sind gescheitert, als sie auf dem Bildschirm erschienen.

Das Christentum hat etwas zu bieten, nicht einmal so wenig: eine Botschaft, die es selbst - früher mehr als heute - eine Frohe Botschaft nennt, mit Erlösung und Befreiung als zentralen Punkten. Gerade in Zeiten, in denen Idh-sucht, Unbekümmertheit und Katastrophendenken stark im Vordergrund stehen, ist dieses Gegengewicht 
beachtlich: Freude, falls sie gut vermittelt wird, hat einen Nachrichtenwert. Das Evangelium kann eine Dauer-Show an Guter Nachricht sein.

Chancen übergenug für die Kirchen, die Medien zu benutzen, um die Menschen zu erreichen, von denen so viele seit Jahr und Tag keine Kirche mehr von innen gesehen haben, die aber doch darauf warten, in ihrem Christensein angesprochen zu werden und die auch bestrebt sind, danach zu leben. Hier sind Chancen, eine Aussicht zu bieten auf ein freudvolleres Leben und hinzuweisen auf Verantwortlichkeiten und die Möglichkeiten, die es zu verwirklichen gilt. Abschieben auf die Kirche ist eine Modeerscheinung geworden. Man erschrickt über die vielen, die mit einem Trauma aus ihrer römisch-katholischen Erziehung herumlaufen. Und doch sprechen andere mit einigem Heimweh von früher, als man wirklich noch zu feiern verstand. Die Frage darf gestellt werden, ob das immer so war, denn die heidnischen Katastrophendenker von heute finden ihre großen Vorgänger in den Kirchenführern und Donnerpredigern von ehedem. Selbst dem düstersten Unheilspropheten der achtziger Jahre würde es nicht einfallen, andere anzusprechen, wie es ein Bischof von Breda im vorigen Jahrhundert tat: „Hört, fleischlich gesinnte, alt gewordene Sünder, und erbebt; hört, weltliebende, schlaftrunkene Christen, und wacht auf " .

\section{III}

Es gibt einzelne Pfarrer, die ihren großen oder kleinen Unmut äußern über das, was Zeitung, Rundfunk und Fernsehen bringen. Abgesehen davon, daß sie bestenfalls einmal Recht haben können ("eine Zeitung ist auch nur ein Mensch“, sagten wir früher in Fachkreisen), bedienen sie sich einer Form der Meinungsäußerung, die immer wieder vorkommt: der Verallgemeinerung.

Ein einziger unrichtiger Bericht, und die ganze Presse taugt nichts. Einmal der Klatsch eines sogenannten Boulevardblatts, und "die Journalisten bauschen nur auf“. Und wie oft hört man, daß das Fernsehen gegenwärtig wertloser sei als früher, und so werden leichthin alle möglichen guten Programme, selbst wenn sie tatsächlich vielleicht seltener sind als einst, nicht mehr beachtet.

Das große Problem dieser Pfarrer aber sitzt tiefer. Abgesehen von einigen jüngeren unter den Pfarrern, sind sie in einer Kirchenkultur erzogen und ausgebildet, die einen ausgesprochen geschlossenen Charakter hatte: In einer Dorfkirche, mit dem Pfarrer als beherrschender, ausschlaggebender Gestalt. Hinzu kam eine eigene sklavisch hörige katholische Presse. Darüber ist allmählich genug geredet und geschrieben worden. Die Kommunikation war eingleisig und bestand als eigentliche Kommunikation nicht, auch wenn man die Bedeutung der Kirche für den Empfang von Information durch die Kirchgänger nicht bestreiten kann ${ }^{8}$.

Als sich die Situation wandelte und die sechziger Jahre mit ihrem Ruf nach Mitspracherecht kamen und die Menschen freimütiger zu sprechen begannen, erwies sich ein großer Teil der - auch der älteren - Pfarrer als bestens vorbereitet, hier mitzutun. Ihre geistige Anpassungsfähigkeit war und ist beachtlich. Das Fernsehen, aber nicht weniger der allgemeine wachsende Autobesitz öffneten das Land, brachten die entlegensten Winkel in den Blickpunkt des Interesses und sorgten dafür, daß jeder über jeden alles wußte - auch kirchlich, und zwar mit allen Folgen. Zeitungen fusionierten notgedrungen und gaben ihren ausschließlich christlichen Charakter auf, oder sie mußten, um der Auflage willen, auch Berichte über andere Kirchen und Parteien bringen. 
Nicht immer begriff man, daß auch ein uraltes Medium gründlich seine Form verändern kann und so brandneu werden kann. Die heutige Berichterstattung der Zeitung, wo leider immer wieder Nachrichten und Kommentare miteinander verwoben werden, ist völlig anders als vor 25 Jahren.

Zunächst besteht in den Niederlanden die partei- oder gruppengerichtete Tageszeitung kaum noch. „Het Vrije Volk“ ist sozialistisch, ist aber weder an eine Partei noch an eine Gewerkschaft gebunden, die früher das Rückgrat bildeten. Die Tageszeitung "Trouw" ist nicht christlich, aber immer weniger ausschließlich protestantisch. Auch ein linksgerichteter Pastor darf sich äußern. Die Breitenwirkung der Rundfunkanstalten bringt es außerdem mit sich, daß ein Zeitungsleser nicht länger Meinungen aus einer einzigen Küche vorgesetzt bekommt. Die Diskussionen in den Parteien über Kernwaffen in den Niederlanden werden überall ausführlich abgedruckt. Allen möglichen Politikern wird oft $\mathrm{zu}$ einer Bekanntheit verholfen, die ihnen vielleicht aber auch nicht zukommt.

Drohte früher die Gefahr einer zu engen Bindung an eine Partei oder Gewerkschaft, so ist es augenblicklich die Art und Weise in der man sich Eingang bei den Medien verschaff $t$, in der jemand "Nachrichten " zu machen versteht, indem man als Politiker mit dem Fahrrad fährt, die Frisur verändert usw.

Oberdies ist die Art und Weise der Befragung, die Interviewtechnik, völlig anders geworden. Wurde in den sechziger Jahren gesagt, daß man einen Befragten bisweilen vor sich selbst beschützen mußte, so geht es augenblicklich eher darum Fallen zu stellen, wenngleich die meisten Zeitungen noch durchaus ihre Maßstäbe haben für das, was sie bringen, und vor allem das, was sie nicht bringen. Doch unter dem Einfluß des Fernsehens ist der Ton und die Art viel schärfer und auch kritischer geworden. Und oft wird vom Journalisten auch mehr Fachkenntnis verlangt, wenn er bei einem Fachgespräch in der Lage sein will, mit Erfolg auf Antworten einzugehen. Die Spezialisierung ist überall vorgedrungen. Der Alleskönner existiert nicht mehr.

Ein Beispiel für die gewandelten Zeitungsnachrichten liefert die Sportrubrik. Nicht nur wird ihr heute viel mehr Raum gegeben, es wird auch anders geschrieben. In den ersten Jahren des Fernsehens kam es vor, daß das englische Cup-Finale am Samstagmittag gesendet wurde und daß eine Zeitung, die erst Montagabend beim Leser in den Briefkasten kam, unbekümmert noch den ganzen Wettkampfbericht brachte. Dies ist heute undenkbar. Jetzt geht die Redaktion davon aus, daß der Interessierte bereits alles durch das Fernsehen und, in diesem Fall vielleicht noch wichtiger, durch den Rundfunk weiß. Die Zeitung bringt also Hintergrund, zum Beispiel Kabinengespräche und Kritik von Spielern an Trainern und so fort. Es scheint, als ob das den Wettkampf bestimmt: bisweilen muß man nach dem Ergebnis suchen.

Die Kirche geriet ebenso hart in dieses Extrem von Interesse für die faszinierenden Veränderungen einerseits, die vor allem von Papst Johannes XXIII. in Gang gesetzt wurden, und von dem Außergewöhnlichen andererseits. Das erste führte zur Polarisierung, denn nicht jeder kann da folgen, und für andere ging es zu langsam; das zweite führte bis zu Meldungen etwa über einen Vogelkäfig oder ein Aquarium in einer Kirche. Während das Publikum einen wahren Durchbruch an Interesse mitmachte, bleibt innerhalb des Rundfunksystems die „Versäulung“ bestehen. Diese Abstufung führte zu einer neuen Situation, die in den Niederlanden einmalig ist. Die einseitige Berichterstattung ist vorbei. Es kommt zum Hören und Wiederhören. Eine Bichofssynode oder eine Papstreise ist schon längst nicht mehr ein selbstverständlicher Alleinanspruch für den katholischen Rundfunk KRO, und eine Diskussion in der Re- 
formierten Kirche über Kernwaffen interessiert alle Rundfunkanstalten: pro und contra, zustimmend oder kritisch, jede Meinung bekommt ihre Chance. Der Zuschauer und Hörer kann alles konsumieren, seine eigene Schlußfolgerung ziehen oder auch in Verwirrung geraten.

Es blieb nicht bei dieser Art der Interessenverbreitung. Auch innerhalb einer Rundfunkanstalt mit lebendiger Überzeugung, wie das einmalige niederländische Rundfunksystem sie kennt, ist es nicht mehr länger möglich, immer denselben Akzent zu setzen. Alte Sicherheiten werden durchbrochen, nicht durch die Medien als Nachrichtenübermittler, sondern durch jene, die die Nachrichten bestimmen. Bis zu den Führungskräften laufen die Auffassungen auseinander. Der Rundfunk weiß nichts Besseres zu tun, als diese Außerungen zu verbreiten mit dem Risiko, abwechselnd als zu links oder zu rechts gescholten zu werden. Eine Rundfunkanstalt mit Prinzip, die sich für eine Mittelstellung entscheidet, ist schon daran, sich selbst zugrunde zu richten. Gerade hier sehen wir als eine Art Gegengewicht in letzter Zeit mehr Bemühungen, sich deutlicher zu profilieren. Jeder sieht alles und spricht über alles oder will den Weg gewiesen haben. Der Pfarrer, von dem wir oben sprachen, wird darauf angesprochen. Wenn die ihm anvertrauten Gläubigen das Ja von der einen und das Nein von der anderer Aktualitätenrubrik gehört haben, wollen sie auch wohl einmal die Meinung des geschätzten Dritten. Bei einer kleiner werdenden Zahl von Kollegen ist er zu einer breiteren Kenntnis gezwungen, überlastet an vielen Enden, wird er gebeten, eine befriedigende Deutung zu geben, in der dann auch noch ein christlicher und gläubiger Ton durchklingen soll. Hier zeigt sich, wie sehr die fehlenden Priester spürbar werden. Es werden nicht viele Priester mehr geweiht, immer stärker vergreist ihre Gesamtzahl, mehr und mehr müssen Pfarreien mit einem Geistlichen zurechtkommen, während früher alle einen Pfarrer und einen Kaplan zur Verfügung hatten. Und die Anforderungen nehmen zu. Will der Pfarrer wirklich in der Kommunikation mitarbeiten, dann tut er mehr als das Menschenmögliche.

Seht, das Neue hat bereits begonnen, können wir mit dem Propheten sagen ${ }^{10}$. Dennoch ist, wider alle christlichen Grundsätze, die Angst vor der Zukunft groß, sogar so groß, daß intern von einem bedrohlichen Alptraum die Rede ist, wo das Evangelium verarmt und verstümmelt weitergegeben wird.

Technisch gibt es wenig mehr zu enträtseln, wenn es um Massenkommunikation geht. Die Worte sind schon da: Video, Bildplatte, Kabel- und Satellitenfernsehen, Glasfaser, Hauskomputer, Visodata, Viditel, Teletext, Neutrino, Kompaktplatte, elektronische Post, Teleshopping, Scribophon. Eine Anzahl dieser Erfindungen wird bereits installiert, wenn auch in bescheidenem Umfang, andere warten auf die Reaktion des Publikums.

Aber lange braucht es ganz und gar nicht zu dauern. In Japan, das auch im Eingehen auf die Praxis vorauseilt, sagt eine ernsthafte Studie folgende Jahreszahlen für den allgemeinen Gebrauch voraus: Einkaufen von der Wohnung aus mit Teleapparat 1991; Wahlen - und zwar direkt, also ohne Wahllokal 1998; Minikomputer in den meisten Wohnungen 1999. Wer heute geboren wird, ist schon, ehe er erwachsen ist, damit vertraut ${ }^{11}$. Das wird zu dem führen, was die Privatisierung der Gesellschaft genannt wird, die man auch als Zellbildung bezeichnen kann, denn ein großer Teil der Arbeit wird zu Hause erledigt werden können. Das Niederländische Postgiro nennt seine Kunden bereits mit einem futuristischen Wort „Hausbankiers", denn der Zahlungsverkehr ohne Münzen oder Geldscheine wird noch zunehmen. So wird auch rasch eine Versammlung über Kabel geschaltet werden. Auch die Schule braucht nicht 
mehr in einem einzigen Gebäude zu liegen. Die neuen Entwicklungen machen technische Zweiweg-Kommunikation möglich: der heutige Einbahnverkehr der Massenmedien wird durchbrochen. Anstelle davon kommt ein Senden, Empfangen und Zurücksenden. Erst das bezeichnet die Verwirklichung einer sozialen Kommunikation ein Wort, das der Vatikan schon lange gebraucht. Sozialer, aber zugleich abgekühlt: Chancen und Gefahren.

\section{IV}

Die Kirche, Trägerin des christlichen Glaubens, wird wohl oder übel mitgehen. Bonhoeffer, so oft schon prophetisch, hat es vorausgesagt: die Welt wird mündig und muß als solche ernst genommen werden.

Zugleich wendet sich die moderne Welt ebensowohl im Prinzip als auch tatsächlich von der Religion $a^{12}$. Der Kern des Problems ist: Wie können Christen, wie kann die Kirche dann auf religiöse Weise so über Gott sprechen, daß die Botschaft in einem Klima verstanden wird, das dafür wenig empfänglich ist? Die Kirche besteht vor allem um der Welt willen, wobei die Kommunikation ihrer Botschaft vom göttlichen Heil und Heilwerden an die Welt ihr Seinsgrund ist ${ }^{13}$. Vorausgesetzt, daß Medien für sich selbst nichts bedeuten, sondern erst Inhalt (gut oder böse) bekommen durch den Gebrauch, den Menschen davon machen, dann muß man sagen, daß die Möglichkeiten heutzutage beispiellos sind, gerade auch für die Verkündigung. Medien sind bedrohend und einengend, aber ebensosehr vielverheißend und bereichernd. Die neue Technik kann Menschen sowohl frei machen als auch ihre Einsamkeit fördern ${ }^{14}$.

Jahrhunderte lang hat man gesprochen von einem Problem des Informationszugangs: wie kommen Menschen in ihren Besitz? Jetzt ist oder kommt das Problem der Informationsverarbeitung. Dazwischen besteht in der Praxis eine riesige Kluft. Von dem Augenblick an, in dem Information nicht mehr verarbeitet werden kann, ist die Sättigung erreicht. Von dem Augenblick an ist es fast unwichtig, wie die Information zu den Menschen kommt: schneller, zweifellos (sogar der Zeitungsjunge mit seiner möglichen Reifenpanne oder Grippe fällt aus), aber darum ist sie noch nicht leichter aufzunehmen. Auch bei besserer und angepaßterer Schulung bleibt eine Grenze für das, was aufgenommen werden kann. Das blitzschnelle Umschalten der Tagesschau ist vielen bereits zu schnell: von einem Krieg in Vorderasien über eine Kundgebung in Zürich und eine Parlamentsdebatte im eigenen Land nach einem Erdbeben in Italien und einem Brand in Rotterdam. Das Tempo, das dank der Technik möglich wird, erweist sich oftmals schneller als die Verarbeitungsfähigkeit des Zuschauers oder Hörers.

Die große Gefahr entsteht, daß der überlastete Mensch eine weitere Wahl trifft und dabei den Weg des geringsten Widerstands einschlägt: sicher über Münz- (also Wahl-) Fernsehen überallhin verbunden wählt er aus einem gewaltigen Angebot das, was ihm paßt: leichte, anspruchslose Arbeit. Einer der schlimmen Ausdrücke unserer Zeit wird allgemein: das entscheide ich doch wohl selbst. „Was ist für uns der Tag? Ein Stück Technik. Wir drehen Knöpfe an und aus. Wir konsumieren einander. Wir konsumieren die Produkte aus den Supermärkten. Wir konsumieren die Erde “15.

Technische Mittel sind im Wesen nicht schlecht. „Wegen der Tatsache jedoch, daß alle Dinge geschaffen sind, haben sie einen eigenen Bestand mit einer eigenen Wahrheit und Güte, haben sie auch eigene Gesetze und Strukturen"18. Es gibt keinen Einwand dagegen, die christliche Botschaft über welches moderne Medium auch immer zu verkünden. Hier haben die Kirchen sich in Sicht auf die Vergangenheit wenig vorzuwer- 
fen. Beim Film waren die Katholiken unter den ersten und in vielen Fällen auch beim Rundfunk.

Insbesondere in den Vereinigten Staaten zeigt sich die Kirche in den Medien wie eine Flut vielverheißender Prediger (Billy Graham), die immer wieder mitteilen, daß die Endzeit begonnen hat (getauftes Katastrophendenken) und daß schnelle Bekehrung geboten ist. Es geht dabei um eine Bekehrung zu einer Form von Glaubenserleben, die sich, bequem, allen Problemen aus dem Weg gehend, von der Welt abwendet.

Dennoch gibt es auch andere Zeichen. In Kanada, so weit wie das Land ist, gibr es einen Gottesdienst über Fernsehen, bei dem Zuschauer sich selbst aktiv beteiligen, auch beim Brechen des Brotes.

Das ist nicht ohne weiteres eine Form des Zusammenfeierns, auf die das Zweite Vatikanische Konzil mit Recht Nachdruck legt, doch ist das unter diesen Umständen eine ernst zu nehmende Sache.

Im Ruhrgebiet, wie wir unlängst im Deutschen Fernsehen sehen konnten, suchen Freiwillige mit Videobändern ans Haus Gebundene auf. In Afrika und Lateinamerika haben christliche Sender dank dem drahtlosen Transistorradio einen größeren Wirkungsbereich. Das Arbeiten mit den Medien ist notwendig und muß sogar verstärkt werden. So muß zunächst verhindert werden, daß kommerzielle und politische Machtblöcke die neuen technischen Möglichkeiten beherrschen ${ }^{17}$, vor allem aber gehört Verkündigung, und zwar mit allen möglichen Mitteln, zum Wesen der Kirche: aus der Priorität des Sendungsauftrags.

\section{V}

Wir leben in einer Welt, die immer kleiner wird. In dem Augenblick, in dem ein Papst in Japan seinen Fuß an Land setzt, können wir es in der ganzen Welt sehen. Es wird sogar so selbstverständlich, daß eine Gewöhnung eintritt und die Möglichkeit zunimmt, daß ein Zuschauer sich durch Knopfdruck aus dem größer werdenden Angebotspaket etwas anderes auswählt. Technisch ist die Welt zwar ein Dorf geworden. Doch die Dorfbewohner werden einsamer. Sie suchen Geborgenheit und ein Wiedererkennen, das zugleich ein Anerkennen bedeutet. Auch auf dem Weg der Medien. Die Lokalblätter gedeihen. Der Ruf nach regionalen Rundfunksendungen nimmt zu. In den Vereinigten Staaten nehmen die großen Tageszeitungen provinzielle Beilagen auf. Viditel, die auf Abruf verfügbare Information über Fernsehen, wird seine Höchstform erreichen, wenn es lokale Information vermitteln wird. Wo nun die Zeiten der Gottesdienste und die Namen der Prediger aufgenommen werden, wird es eingeschaltet. Doch um Menschen zu veranlassen, die Kirche aufzusuchen, ist mehr notwendig, da geht es um Erfordernisse, mit denen die Medien wenig zu schaffen haben.

Mitten in einem riesigen Wirkungsfeld, das kaum weiter reichen kann, sehen wir, wie für viele Menschen ihre kleine Welt zusammenschrumpft.

Im Großen ist internationales und vor allem auch ökumenisches Interesse nötig. Die Christenkirche harrt einer Zukunft als Bewacherin von Werten wie Privatheit und Lebensfreiheit. Sie soll eine Zuflucht sein für die Erniedrigten, Geschundenen und Verlassenen, und Führungskräfte zur Ordnung rufen. Dazu muß sie gehört werden können, auch über die Medien.

Die nicht geringen, an die Erfordernisse der Zeit angepaßten Verpflichtungen müssen ebenfalls erfüllt werden, wenn die Kirche auf örtlicher Ebene angesehen sein will als etwas, das das Dazugehören lohnt. Es geht also auch um eine Stärkung des lokalen Christentums, das Ermutigung nötig hat, wenn es angesehen werden will als „die Eltern im Dorf“. Wo immer der Glaube auch steht, weltweit oder im Miniverband, 
stets wird die Kommunikation wohlüberlegt geschehen müssen, in wechselseitiger Harmonie und unter Einsatz aller verfügbaren Mittel. Darum kann nicht genug getan werden für die Ausbildung und die Bildung, und darum ist eine Rangordnung der Prioritäten notwendig. Für alle in der Kirche arbeitenden Menschen, vor allem aber für die in der Seelsorge Tätigen, ist die Fähigkeit zum Umgang mit den Medien im Bereich auf ihre Möglichkeiten und Eigenheiten ein wesentlicher Teil für die Strategie des Überlebens.

Die Welt ist kleiner geworden, und wir wissen mehr voneinander als je zuvor. Vor allem die Kommunikationsmittel profitieren von den technischen Errungenschaften. Die Kirche hat lange Zeit allein über die gesellschaftliche Kommunikation geherrscht. Jetzt hat sie Mühe, ihre (Frohe) Botschaft zu übermitteln, auch auf lokaler Ebene. Die Technik schreitet weiter fort; die Zukunft hat schon begonnen. Die kirchliche Führung wird alles Augenmerk darauf lenken müssen, als Wächterin über alle möglichen menschlichen Werte und als Kritikerin im Interesse der Unterdrückten, und auch in der Seelsorge auf unterer Ebene muß sie ihre Stimme vernehmen lassen. Das erfordert eine Strategie.

\section{Anmerkungen}

12 Petr 3, 16; Kor 1, 23.

2 Inter Mirifica. Dekret über die sozialen Kommunikationsmittel des Zweiten Vatikanischen Konzils.

3 "De kerk en de massakommunikatiemedia, rapport van de vierde assemblée van de Wereldraad van Kerken “. Ubersetzung C. Hamelink, o. J.

4 "Während seines Erdenlebens hat Christus sich als vollkommener Kommunikator gezeigt. Christi Kommunikation war Geist und Leben $(J o h \quad 6,63) \ldots$ In der Kirche, die der mystische Leib Christi ist, erfüllt $\mathrm{Er}$ es in allem ${ }^{\alpha}$ (Eph 1, 23; 4, 10). Pastoralinstruktion Communio et Progressio.

5 "Christus hat mich gesandt, um das Evangelium zu verkündigen, und dies ist nicht mit "schönen und gelehrten Worten" (1 Kor 19,17). "Siehe mit wie großen Lettern ich euch eigenhändig schreibe ${ }^{\alpha}$ (Gal 6,11). Dieses letzte Wort wird auch wohl ausgelegt als ein Beweis der Echtheit des Briefes.

- Papst Pius XI. (1922-1939) in einer Audienz für katholische Journalisten. Entnommen einem Artikel von J. O. Smit in: „De Heraut van het H. Hart", April 1936.

7 Fastenbrief 1839 von Johannes van Hooydonk, der damals Apostolischer Administrator von Breda war.

${ }^{8}$ H. J. Prakke: Kerkgang om "nieuws ${ }^{\alpha}$. Assen 1965.

- Als der "Vrije Volk ${ }^{*}$-Redakteur Kees Diemer in den fünfziger Jahren einen Streit in der Zweiten-Kammerfraktion der Partij van de Arbeid veröffentlichen wollte, widersetzte sich Chefredakteur K. Voskuil mit der Bemerkung: „Die Dinge bleiben doch wahr, auch wenn man sie nicht in die Zeitung setzt" (Mulder, Arlman, Den Tex: De val van de Rode Burcht. Amsterdam 1980).

10 "Siehe, ich werde etwas Neues machen, es ist bereits am Keimen, wißt ihr das nicht?" (Jes $43,19)$.

11 Wissenschaftliche und technologische Entwicklungen bis zum Jahr 2000, veröffentlicht von der japanischen technisch-ökonomischen Vereinigung 1977. In Titulaer: Toekomstbeeld. Naarden 1980.

12 D. Bonhoeffer: Widerstand und Ưbergabe.

${ }^{13}$ H. Kraemer: Communicatie, een tijdvraag. Den Haag 1957.

14 Erklärung der Medienkommission der Deutschen Bischofskonferenz 1980.

15 Tini Visser: De toespraak. Amstelveen 1981.

16 Pastoralkonstitution über die Kirche in der Welt von heute "Gaudium et Spes ${ }^{\alpha}$.

${ }^{17}$ Hans Florin, Generalsekretär der Weltvereinigung für christliche Kommunikation. In: "Centraal Weekblad" v. 10. Dezember 1980. 


\section{SUMMARY}

Despite the world becoming technically a global village the alienation seems to be bigger than ever. Unlike expectations the communication seems to be converted into the opposite. Everybody is taking care of himself. There are men however who try and fight against this, also from the Christians. But also within the Churches the importance of the media is more adknowledged theoretically than in practice. The Churches are groupings who do need to be part of the media in this world. A theology of communications seems to be necessary. There are still members of the clergy who consider the media as their enemy because of its plurality. The development of the media also entails the danger of a growing privatisation of the society. But the possibilities for proclamation with the media are still great. Therefore all openings for a respective training should be used. The capability to use the media in the Church are definitely important for a strategy of survival.

\section{RESUME}

Bien que le monde se resserre techniquement vers le village, le sentiment d'étrangeté semble plus grand que jamais. Dans un illogisme du développement, la communication semble faire volte-face. Charité bien ordonnée commence par soi-même. Cependant, il y a des gens qui se soulèvent contre cela, en quantité non négligeable parmi les chrétiens. Mais même à l'intérieur des Eglises, on comprend la signification des media théoriquement mieux qu'on ne la met en pratique. De nos jours, les Eglises font partie des groupes qui doivent se faire une place dans les media. Une théologie des mass media est necessaire. Malgré la faculté d'adaptation du clergé, il y a encore des prêtres qui voient dans les media un ennemi parce que chaque opinion a une chance. Le développement des media entraîne avec lui le danger d'une privatisation croissante de la société. Cependant, les possibilités d'une annonciation, également à l'aide des media, sont très grandes. Pour cela, on doit utiliser tous les moyens pour une formation correspondante. La faculté d'utiliser les media est, dans l'Eglise, particulièrement importante pour la stratégie de la survie.

\section{RESUMEN}

Aunque la técnica convierte el mundo en una aldea, nunca fué mayor el distanciamiento entre los hombres. Hay en el desarollo un proceso ilógico que convierte la comunicación en su contrario. Cada cual es su prójimo. Sin embargo hay personas, y no son pocas entre los cristianos, que se resisten a este proceso. Pero incluso dentro de la Iglesia se capta mas teórica que prácticamente la importancia de la comunicación social. Las iglesias forman parte hoy de los grupos que aún deben conquistar un lugar en los medios de comunicación. Es necesaria una teología de los medios. A pesar de la capacidad de adaptación del clero, todavía hay eclesiásticos que ven en los medios un enemigo porque dan una oportunidad a cada forma de pensar. El desarollo de los medios comporta el peligro de una creciente privatización de la sociedad. Sin embargo son muy grandes las posibilidades de proclamación a través de los medios. Por ello deben aprovecharse todos los recursos para una formación adecuada. La capacidad de manejarse con los medios es importante en la Iglesia como estrategia para sobrevivir. 